\title{
Sub-0.6 dB NF Low Power Differential to Single-Ended MMIC LNA Designs for the SKA using InGaAs/InAIAs/InP pHEMTs
}

\author{
S. Arshad ${ }^{\star}$, B. Boudjelida, S. Boulay, N. Ahmad, M. Missous
}

School of Electrical and Electronic Engineering, University of Manchester, UK.

\begin{abstract}
The need of a highly sensitive system in SKA necessitates the development of ultra low noise, high gain yet low power LNAs for use at the front-end of the receiver chain. The input interface of the LNA has to be differential to be able to integrate with the receiving antenna while the output must preferably be single-ended matched to standard $50 \Omega$ system impedance. This work presents a design for a low power, differential-single ended LNA for the SKA. The simulated results show that the LNA achieves $<0.6 \mathrm{~dB}$ Noise Figure for the wide frequency band of $300 \mathrm{MHz}$ to $1 \mathrm{GHz}$ at room temperature. The forward gain, at around $30 \mathrm{~dB}$, is fairly flat for the required frequency range. The simulated power consumption of the designed LNA is approx $300 \mathrm{~mW}$ which is $1 / 3 \mathrm{rd}$ of the power consumed by similar LNA realised using commercially available GaAs pHEMTs. Design layouts are being prepared for the fabrication of these LNA circuits.
\end{abstract}

\section{Introduction}

The Square Kilometre Array (SKA) is the largest radiotelescope project presently being designed that is will be up to 100 times more sensitive as compared to any existing radio telescope (Hall et al. 2004) . This feature demands high end performance from the LNA in terms of noise figure and gain. The magnitude of the envisioned radio telescope, on the other end, poses highly challenging requirements towards the power constraints of the constituting components. A differential input and single-ended output is needed from the LNA for interfacing with the receiving antennae at the input and subsequent stages of the receiver chain at the output.

An InP pHEMTs based double stage, differential to singleended MMIC LNA design is presented in this work. The designed LNA is based on novel InGaAs/InAlAs/InP pHEMTs that has been developed and fabricated at the University of Manchester (Bouloukou et al. 2006). The stacked layers of pHEMT used in this work is presented in Fig. 1. The linear and non-linear models for the pHEMTs used in this design have been presented in Boudjelida et al. (2008). In order to verify the complete LNA design and development process, a single stage LNA, based on a $1 \times 800 \mu \mathrm{m} 2$ periphery gate transistor has been designed, fabricated and measured across the wide frequency band of 0.3 to $3 \mathrm{GHz}$. The design and fabrication of this single-stage LNA was based on measurements and modelling of all single elements in the circuit, namely, pHEMTs, inductors, capacitors and resistors. A detailed description of the design and measured results for the single-stage MMIC LNA is being presented in a parallel paper on low frequency broadband LNA designs for the SKA (Boulay et al. 2010).

\section{MMIC LNA Design Process}

The MMIC LNA designs, based on the latest InGaAs/InAlAs epitaxial structures, chosen for this work had more emphasis on

^Shahzad.Arshad@postgrad.manchester.ac.uk

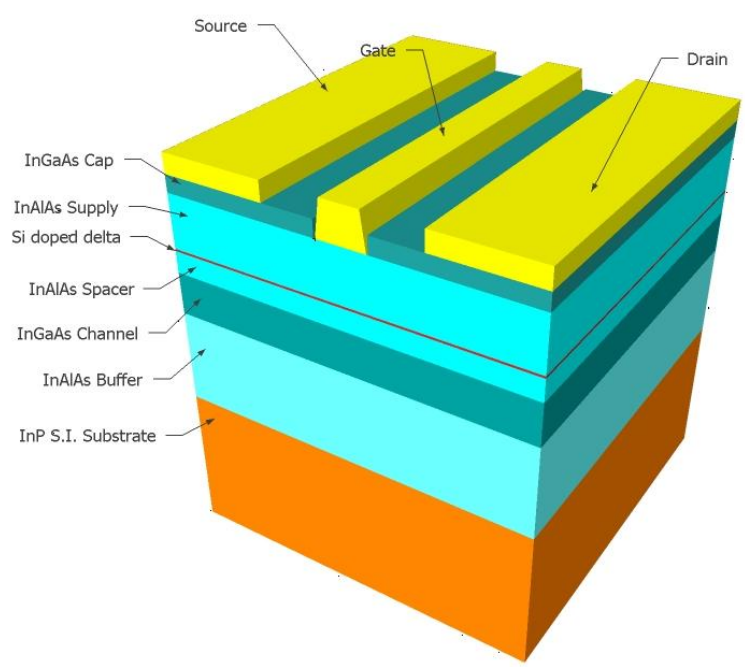

Fig. 1: Stacked epitaxial layers of pHEMT

low noise figure and low input and output return losses across the wide frequency band of 0.2 to $2 \mathrm{GHz}$. To accommodate these design requirements, the design process is based on $1 \mu \mathrm{m}$ gate length InGaAs/InAlAs/InP technology with varying gate widths.

Coplanar circuits have been used in this work for the implementation of MMIC LNA designs which has several advantages over the conventional micostrip circuits, a few prominent are as follows (Gillick et al. 1994):

- Devices and components can be grounded without via holes, as the ground plane lies on the same surface.

- Dispersion in CPW is much lower at high frequencies, making it more suitable for millimeter-wave circuits.

- Flexibility in realising given characteristic impedance with almost any track width and gap combination. 


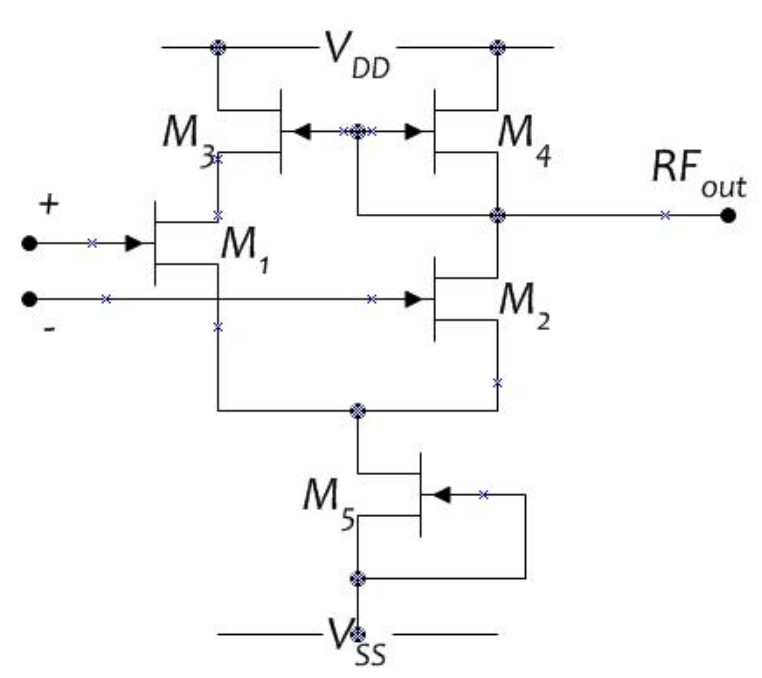

Fig. 2: Standard symmetrical arrangement topology for differential to single-ended amplifier

- The ground plane provides shielding between adjacent CPW lines, which considerably increases the packaging density of the components.

- With the back-face ground plane removed, lumped elements exhibit less parasitic capacitance.

- A well established on-wafer measurement technique based on coplanar probe tips is commercially available, facilitating easy and accurate measurements.

For the selected design process, it has been demonstrated that CPW can be used at these frequencies for low noise designs and yet still meet size limitations necessary for low cost production. The attractive features of this process include reduced back side processing, no vias, and suitability for flip chip mounting.

\section{Double-Stage Differential to Single-Ended MMIC LNA Design}

The requirement of differential input LNA in SKA is to be able to interface with the balanced terminals of the antenna. The output interface, however, has to be single ended with $50 \Omega$ output impedance. A differential to single-ended LNA has thus been designed to meet both the input and output requirements. For a differential to single-ended conversion, half of the gain achieved by the differential stage is lost since each arm effectively only amplifies one half of the applied differential input. Several topologies have been reported that overcome the loss in gain (Ladbrooke 1989). For this work, the standard arrangement for differential to single-ended amplifier shown in Fig. 2 has been used.

This design comprises two stages where the first stage is fully differential, optimised for noise performance with reasonable gain, while the second stage is a gain and stability boosting stage with differential input and single-ended output interfaces. The noise performance of the first fully differential stage
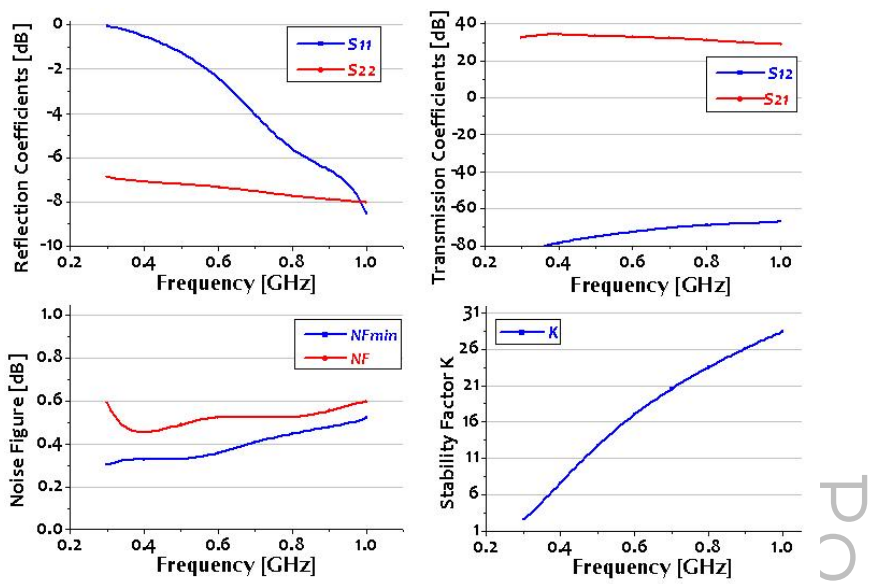

Fig. 3: Simulation results for double stage differential input singleended output MMIC LNA design

is considerably better as compared to the second differential to single-ended conversion stage due to the balanced topology. Due to the considerable high gain of the first stage (12-15 dB), the relatively degraded noise performance of the second stage has minimal effect on the amplifier's noise characteristics, as expressed by the Friis' expression for noise figure of cascaded microwave networks (Friis 1944).

The designed LNA is optimised for noise figure and power consumption with a fairly high gain of $30 \pm 2 \mathrm{~dB}$ for the low frequency band of SKA (0.3-1 GHz). A noise figure of less than $0.6 \mathrm{~dB}$ with unconditional stability for the entire frequency band of interest is predicted for this design with power dissipation of $300 \mathrm{~mW}$. The simulated results presented in Figure 7 show high losses at lower frequencies for the input because of the absence of input matching network. Reasonable output SWR S22 and good reverse isolation S12 are predicted for the design. Layouts and masks are currently being prepared for this design.

\section{Conclusion}

The differential to single-ended MMIC LNA design presented in this work has several advantages over similar MIC differential to single-ended LNA using off-the-shelf GaAs pHEMTs. Foremost of these advantages is that this MMIC LNA consumes less than half power $(300 \mathrm{~mW})$ as compared to the power consumed by a similar MIC LNA design $(800 \mathrm{~mW})$. This has only been possible because of the freedom of using devices with smaller gate widths in the MMIC LNA design while the MIC LNAs have limited options of gate widths available to be used in the designs. In a practical scenario, where millions of LNAs would be employed, the low power characteristic will be crucial. For mass production of LNAs, which will be required in the SKA, MMIC will be a preferred choice for incurring lower cost on production as compared to hybrid MIC LNAs. A reliable and verified design process has been selected for the implementation of this LNA design.

Realising a high performance, small size, reliable, low cost and low power MMIC LNA with differential input and 
single-ended output interface is one of the key concerns for the SKA and a reasonable effort has been done here to meet these requirements. The MMIC LNAs based on $1 \mu \mathrm{m} \mathrm{InP}$ based pHEMTs have high potentials for use in the SKA.

\section{References}

Boudjelida, B., Sobih, A., Arshad, S., Bouloukou, A., Boulay, S., Sly, J., Missous, M., "Sub-0.5 dB NF broadband low-noise amplifier using a novel InGaAs/InAlAs/InP pHEMT" in 7th International Conference on Advanced Semiconductor Devices and Microsystems. 2008. Smolenice, SLOVAKIA: Ieee.

Boulay, S., Boudjelida, B., Arshad, S., Ahmad, N., Missous, M., "Novel Ultra Low Noise Amplifier based on InGaAs/InAlAs pHEMTs," 2010, in Proc. Wide Field Science and Technology for the SKA, Limelette, Belgium, S.A. Torchinsky et al. (eds), ASTRON, ISBN 978-90805434-5-4

Bouloukou, A., Sobih, A., Kettle, D., Sly, J., Missous, M. "Novel High Breakdown InGaAs/InAlAs pHEMTs for radio astronomy applications" in Proceedings of the 4th ESA Workshop on Millimeter Wave Technology and Applications (7th MINT Millimiter-Wave International Symposium). 2006. Espoo, Finland.

Friis, H.T., Noise Figure of Radio Receivers. Proceedings of the IRE, 1944. 32(7): p. 419-422.

Gillick, M., Robertson, I.D., Aghvami, A.H., "Uniplanar techniques for monolithic microwave integrated circuits" Electronics \& Communication Engineering Journal, 1994. 6(4): p. 187;194.

Hall, P.J. The square kilometre array: An international engineering perspective. in Square Kilometre Array Meeting (SKA). 2004. Penticton, CANADA: Springer.

Ladbrooke, P.H., MMIC design : GaAs FETs and HEMTs. The Artech House microwave library. 1989, Boston, Mass., London: Artech House. 370. 